\title{
How to Develop a Leadership Training Program
}

\author{
James W. Fleshman, MD, FACS, FASCRS ${ }^{1}$ \\ ${ }^{1}$ Department of Surgery, Baylor University Medical Center, Roberts \\ Hospital, Dallas, Texas \\ Clin Colon Rectal Surg 2020;33:204-211.
}

\author{
Address for correspondence James W. Fleshman, MD, FACS, FASCRS, \\ Department of Surgery, Baylor University Medical Center, 3500 \\ Gaston Avenue, 1st Floor, Roberts Hospital, Dallas, TX 75246 \\ (e-mail: james.fleshman@bswhealth.org).
}

\section{Abstract \\ Keywords \\ - leadership \\ - training \\ - characteristics \\ - culture}

Leadership training is an essential component of faculty development and resident training. Characteristics of leaders include growth mindset, curiosity, humility, selflessness, intrinsic motivation, hunger to achieve, insight, collaboration, harmony, introversion and analytical approach (inherited) and emotional intelligence, empathy, flexibility, adaptability, conflict management, resilience, interpersonal skills, and judgment (learned). Training for each of these characteristics will enhance the leadership abilities of the surgical department.
Leadership was, in the past, an undefined and existential quality sought after by institutions and departments of medical specialties that could only be gained by hiring in "leaders." Recently, business schools have begun teaching leadership and a number of publications about leadership have increased over the last decade. Unfortunately, medical education has not caught up with business in this effort to train leaders. Rather many institutions continue to rely on happenstance to find leaders in health care specialties. Surgery as a specialty has started training leaders at a national level, with special courses available through the American College of Surgery for fellows of the college. Individual departments of surgery have an opportunity to create a culture of leadership and develop leadership training within the department for all members of the department, regardless of the experience of the individual member. This provides the opportunity to shape careers, enhance function within the department, utilize innovation, and improve care for our patients because we can enhance or capture the potential of each member of our department of surgery. What follows is the why, who, when, what, and how to create a leadership program in a department of surgery based on our experience at the Department of Surgery, Baylor University Medical Center in Dallas.

\section{Why?}

1. Surgeons need leadership skills in numerous areas:

(a) Operating room teams, PODs, and committees. The smooth, team-oriented function of an operating room and the governance of an operating room POD (operating room team grouping) will only exist if the members of the team are familiar with the leadership characteristics of self-awareness, vision, and intrinsic motivation. The collaborative efforts of all involved in the care of a patient require willingness to sacrifice ego and selfperceived job descriptions. No hierarchical authoritative organization needs to exist in a collaborative atmosphere where all members of a team accept responsibility for the success of the procedure for each individual patient. In this circumstance, there can be no limit to the responsibilities of the leader, who will lead most effectively from the center of the group as a shepherd as opposed to an authority. This does not remove the ultimate responsibility for the success of operation from the surgeon, rather guarantees more interested parties focused on the overall success.

(b) Resident teams function to provide consistent care. The chief resident or senior resident in charge of a team has the opportunity as a leader to instill a vision of patient care and provide guidance to those junior team members and allied health care associates who look to them for guidance and experience derived judgment. Teaching on a surgical service becomes more than passing on medical knowledge.

(c) Nursing floors as medical director or managing care of patients. Physicians who assume leadership roles often have not had training in leadership skills or even the fundamental elements of business management and educational leadership. Leadership, in these
Issue Theme Leadership; Guest Editor: James W. Fleshman, MD, FACS, FASCRS
Copyright $\odot 2020$ by Thieme Medical Publishers, Inc., 333 Seventh Avenue, New York, NY 10001, USA. Tel: +1(212) 760-0888. ISSN 1531-0043. 
areas that cross boundaries of disciplines, requires special attention to respect and inclusiveness to develop a collaborative team for patient care.

(d) Educational and administrative roles in the training program. As surgeons grow in experience and responsibilities, there are essential elements of behavior and personal interaction which will enhance their ability to develop teams and guide those groups that follow them in their care of patients. In most medical schools, this is not a required component of the curriculum. Residency training programs require different levels of training and involvement from faculty to create an atmosphere of curiosity, accountability, mentorship, scholarship, and sacrifice.

(e) Societies at local, regional, and national levels. In academic and private practice, surgeons have an opportunity to change our work world and influence many more individuals, including patients, outside of the population where we spend our daily effort. Belonging to a professional society does not guarantee a leadership role. Only after a great deal of volunteer, selfless effort, will leadership roles arise. Leadership skills are essential to function in this highly political world. Without training in leadership, a surgeon can fail to realize their potential when offered a leadership role.

(f) Administrative positions within practices, hospitals, systems, industry partners, and insurance companies. In any setting, there are roles which require leaders. A surgeon can only be successful as a leader if trained to function at a high level of collaboration and recognize the need for specific nonmedical skills. Understanding business practice is essential for administrative decision making. Even after business training, leadership skills remain an extra component of training to allow the surgeon to be effective in administrative positions. Interpersonal skills and self-awareness are often taken for granted at a leadership level. Leadership training provides the surgeon a vocabulary and awareness of senior leadership functional requirements.

(g) Medical School, Departmental, Practice, and Hospital Committees. Promotion to leadership levels and decision-making positions in medical institutions require selfless motivation aligned to the vision, mission, and values of the institution. Unless the leader can subjugate their own needs to the institution's best interest, they will be an ineffective leader. Learning an approach to creating alignment in oneself and others, driving a vision for change or asking others to make sacrifices is essential to successful leadership.

(h) Different leadership approaches yield skills that solve different problems. In most institutions, the environment provides opportunity for problem solving and innovation. The ideas and needed changes are usually already known, available, and clear, but implementation is difficult. Leadership training not only provides the techniques to put these changes in place but may provide an alternative answer to the problem. The ability to look at problems from a different and sometimes nontraditional direction can be taught in leadership training.

2. A leadership culture raises the bar. The culture of an institution depends on the values and mission of the people within it. Changing the culture to one in which participants feel themselves capable of being a leader, willing to take on change projects, and support the institution and those that it serves, can only be beneficial. The building of teams based on leadership skills can only enhance the ability of the institution to achieve goals The results of leadership training are the following:

(a) Expectations for all to contribute at a very high level will actually result in superior results. Personal commitment to a task at hand becomes common place and very little effort needs to be expended to create alignment.

(b) Quality feedback for all team members becomes the standard and allows everyone to improve and reach their potential. High potential leaders are able to rise to the fore in a culture of leadership.

(c) Emotional intelligence is taught and incorporated into daily life when leaders realize the basis of trust is empathy and self-awareness.

(d) Conflict management changes to self-management because the desire for a win-win-win solution is foremost for all involved in the conflict. Conflict is inevitable in a busy, forward moving system which is creating change. The use of conflict to create new and interesting solutions is only possible when everyone involved understands the principle of open dialog and consideration of all options before making the best decision.

(e) Insight is gained from empathy applied to all interactions and reduces the need for negative reinforcement or punishment. Leaders create options for incentivizing team members to give their best.

(f) Practicing leadership principles produces a positive response in coworkers who are leaders themselves.

(g) Leadership knowledge generates special projects which are complex and have high potential for good outcome above and beyond the routine. Team members who understand leadership are willing to commit and understand the impact of self-sacrifice on success.

(h) Continuous education and improvement becomes routine when leaders are contributing at the optimal level, as part of a senior leadership team.

(i) Coaching and mentoring becomes part of daily life and does not require coercion. The ability to mentor becomes an outcome measure for leadership. Coaching for members of the leadership team can enhance the function of executives or surgeon leaders.

(j) Intrinsic motivation develops in team members. The intangible rewards of leadership become the motivation to take on more leadership roles and make more and more sacrifices for the team or institution. 
(k) Different leadership approaches learned during training may yield new unused skills that solve problems in an unexpectedly beneficial way.

3. A culture of leadership stimulates:

(a) Innovation arises from trained leaders having intrinsic motivation and understanding the benefit of collaboration.

(b) Research becomes a natural next step for leaders in many different areas of life and medicine as ideas are generated to solve problems and make improvements in patient care.

(c) Involvement in education becomes a natural extension of selflessness and a motivation for transfer of knowledge to team members.

(d) Quality improvement has origins in leadership out of hunger to contribute.

(e) Leaders seek and practice mentorship from the desire to identify and create other leaders.

(f) Alignment to the hedgehog concept (the one most important thing that the institution can do for people) becomes the driving force behind the team.

(g) Selfless level 5 leadership (the highest level of leadership) begins when the individual seeks success for all members of the team.

(h) Leadership training results in the attraction and recruitment of high potential team members who strive for leadership themselves and become high-level producers.

4. Leadership is based on:

(a) Vision: A clear definition of success and a pathway to accomplish success.

(b) Flexibility: Willingness to try new processes and different pathways to success.

(c) Selflessness: Credit, congratulations, and reward are not necessary for continued contribution.

(d) Motivation: Intrinsic (self-determined without external enticement) motivation is adequate to push the leader to greater heights.

(e) Emotional intelligence: Self and social awareness allow the leader to function at a high level in critical, high pressure, politically charged situations.

(f) Empathy: Recognition of the emotional state of self and others gives needed info to the leader to allow quality feedback, consensus, collaboration, and motivation of others.

(g) Adaptability: When circumstances change beyond control, the leader is able to switch to and embrace an alternative pathway to success.

(h) Resilience: Able to accept and utilize failure to provide motivation to move forward to success.

(i) Growth mindset: Continuous search for new ideas and willingness to change indicates a flexibility and adaptability necessary for leadership

(j) Confidence: Trust in the ability of the team and oneself to accomplish all that is needed to achieve success.

(k) Reliability: Able to be present and functioning when needed to bring stability to the team.
(1) Accountability acknowledges and takes responsibility for personal and team failure to bring positive to a negative situation.

(m) Influence: Develops relationships which can be used to change behavior and bring resources to achieving a desired goal.

(n) Judgment: Decision making based on experience allows the leader to assess and manage a situation with good outcomes, choose between options and develop strategy.

(o) Managing: Pulling the right people together as a team and providing guidance, resources, feedback, and time awareness to achieve prescribed goals.

(p) Directing: Focusing team members on the vision, goals, and objectives to create success

(q) Engaging: Providing continuous encouragement, positive reinforcement, and opportunities for team members to be completely involved in the desired outcomes and be successful.

(r) Interpersonal skills: Learning emotional intelligence guides the personal interaction with team members to support the function of the team.

(s) Ability to give and receive feedback: Accurate and appropriately delivered positive and negative real-time feedback is essential for team members and leaders to accomplish continuous improvement in any situation.

5. A leadership culture reduces the need for management of department members:

(a) Ideas are forthcoming from other aligned and engaged team members based on good judgment, selflessness, accountability, adaptability, and flexibility.

(b) Process issues are forefront in a leader's mind and the team members who are functioning at every leadership level require little management to identify appropriate pathways to success.

(c) Makes everyone think at a higher level reinforced by emotional intelligence, common vision, alignment, and the values of the institution.

(d) Data become the source of ideas and feedback and measure progress, not the leader. Self-governance and autonomous determination are intrinsic motivation factors that induce positive reinforcement to the individual and reduce the needed input from the leader.

(e) Provides another method to reward team members by placing them in leadership positions. Opportunities to lead should be distributed in a positive way to indicate they are being awarded as a reward.

\section{Who?}

All members of the department should be considered as candidates for leadership training:

1. Faculty: Whether private practice, employed or academic, full-time or part-time

2. Fellows: Regardless of specialty

3. Residents: At all levels 


\section{Students}

5. Staff and administration.

The integral group of educators for creation of the leadership training program includes the psychologist who lives in the leadership space, the residency training program director, the division chiefs of specialties within the department of surgery (including the division of surgical education), the senior leadership team within the department of surgery and the department chairman. The decision to move forward with training in surgical leadership was made by all of these individuals as part of the department strategic planning process.

\section{When?}

1. Seminars for the faculty within the department are set at a time which is already set aside as an educational time period, thus preventing further time commitment on the part of the participants.

(a) Six seminars each 2.5 hours long

i. Developing physician leaders for successful clinical practice

ii. Know thyself-leadership and personal values

iii. Ten common mistakes in leadership

iv. Leading team effectively and how to manage difficult team members

v. How to manage demanding situations and decisions-conflict management

vi. Change management-How to effectively lead a cultural and organizational change (Part 1), flourishing in the workplace as a leader (Part 2)

(b) During time already set for conferences (to reduce encroachment into faculty time personal or productivity time)

(c) By invitation only with set expectations, to create the reward mindset key to creating leaders

(d) Start with junior faculty and then mix in senior faculty to build a group of faculty with a vocabulary of leadership.

2. A series of six leadership seminars held monthly off-site at a local university are designed for health care leaders to receive a certificate of training and serve as a prerequisite for appointment to committees of the medical center and employed physician model.

3. A year-long off-site training at an executive health care MBA program, held on weekends, allows faculty to receive a degree in 1 year.

4. Departmental grand rounds are designed to cover professionalism, integrity, and ethics (PIE rounds) for residents every other month for six sessions which will cover the above set of topics in a didactic session with discussion about relevance to their function as residents.

\section{What?}

1. Define leadership

(a) Meaning: A leader has followers to accomplish a common goal. (b) Types: Leadership occurs with variations in motivation, projection of self, expectations of team members, use of emotional intelligence, communication skills and styles, reliance on authority or influence, and emphasis on self-sacrifice.

(c) Metrics: Success is measured not only by immediate success but more importantly by sustained success even after the leader has moved on to another part of life. While the end game is a primary focus of outside observers, the means of achieving the goal is probably more important since the continued relationship of the team allows ongoing success in other tasks. The leader has several functions which can be measured using the Fisher's integrative leadership grid.

(d) Characteristics: There are characteristics of leaders that can be developed and others that tend to be inherent in the individual. Growth mindset, curiosity, humility, selflessness, intrinsic motivation or hunger to be achieving and driven, insight, a concern for collaboration and harmony, introversion, and analytical approaches to decisions are personality characteristics that are inherited and difficult to change. Emotional intelligence, empathy, flexibility, adaptability, conflict management, resilience, communication and interpersonal skills, and judgment are characteristics that can be learned. Many leaders in the past were fortunate to accumulate positive characteristics unintentionally. Leadership training is available to help an individual develop missing attributes.

2. Noncharacteristics of leaders are as follows:

(a) Charisma: Projection of oneself onto the group can be helpful to get started as a leader but relying on the force of personality to lead a group can be detrimental to the strength of the group and prevents the individuals in the group from believing in themselves to be leaders and contributors.

(b) Extroversion: The need to have others provides the energy and motivation for commitment to a program can be problematic in a change culture. Extroverts not only project themselves but they also need affirmation. While this can be unlearned, it is difficult to maintain a sense of meaning and motivation in a setting where little recognition is available.

(c) Ego: Most executives have an ego that is resilient and able to handle negative feedback. However, it is apparent that a belief in infallibility can lead to ignoring the voice of reason or dissention that carries real insight and warning.

(d) Physical size and athleticism: Evolution has placed the idea that a tall imposing figure is needed for leadership. In a military setting or in a tribal community, physical presence may be beneficial, but in today's world, and certainly in surgery, height and physical presence has little significance. Coordination and dexterity are key to surgical skills and therefore provide the backdrop to respect for a surgical leader but are not essential. 
(e) Superior intellect: In a profession where a standard of education and continual learning are required, the differential that a superior intellect brings to leadership is minimized. As in other areas of leadership, emotional intelligence far outweighs intellect.

3. Experiential is as follows:

(a) timonials from leaders: The best evidence for providing incentive for team members to undergo training for leadership is the personal testimony of leaders who understand the need for extra training in the leadership skills. A question and answer period led by an experienced educator can bring out the salient points for the training group.

(b) Debriefs of real situation: The best opportunity for learning can be found in situations in and around the institution which are relevant to each of the team members. The analysis of the issues and the performance of the principles in the situation can be very instructional to all of the team members as they recognize their potential roles in the event or decision.

(c) Practical methods of developing leadership are:

i. Assign team members to leadership positions. The hierarchical structure of a department can be used to provide opportunities for leadership at all levels of experience and training and the result should be experience level appropriate opportunities. The chief and deputies of the department or division can utilize every task that arises to promote the leadership skills of the members of the unit.

(1) Division and section chiefs for each specialty

(2) Coordinator roles in student, resident, and faculty education

(3) Tutorial leaders for students

(4) Residency training program director and assistant directors

(5) Case management conference leader for each specialty

(6) Research director for each specialty area

(7) Representative of the section, division, or department to the cancer center

(8) Representative to cooperative group trials

(9) Lead physician for value-driven efforts in the department (enhanced recovery after surgery, centers of excellence, research group)

(10) Director for continuing medical education

(11) Director for outreach efforts for each service line

(12) Medical directors for each service line and nursing unit involving the department

(13) Quality improvement teams in the department

(14) Hospital committee appointments from the department

(15) Operating room block time committee representative
(16) Operating room POD leaders in each specialty and subcommittee leaders for specific projects

(17) Representatives of the department to the national societies from the different specialties to encourage membership and abstract submission to the national meeting

(18) Standardization of care leaders and POD leaders for the operating room.

ii. Create teams to manage departmental issues. Strategic planning for the department typically illuminates areas of need for planning and focus. These resulting projects always present opportunities for participation by the members of the department and require leadership.

(1) Newsletter group

(2) Annual report group

(3) Research publications group

(4) Mentorship group

(5) Resident education group

(6) Faculty development group

(7) Research peer-review group

(8) Simulation group

(9) Surgical education group

(10) Outreach group

(d) Understanding styles of leadership are:

i. Transactional-directional management with no subordinate input. Leader expects commands to be followed without question. Command and control leadership based on financial or decision control.

ii. Hierarchical-based on the authority of the position and the underlying leadership grid supported by the institution.

iii. Transformational-an approach to change which requires buy-in from the entire team and a leader who considers all input from team members and team approval as criteria for success. Transformational leadership takes advantage of team member ideas and willingness to take them to completion as part of the overall strategy for the department.

iv. Shepherd-the leader guides, protects, and directs from behind the team members of the group and advises as they find their way to success, without leaving any members behind. The leader looks for the best opportunities for the group to pursue and then allows the group to be successful.

v. Servant-the leader considers their key role as supporting the team in all their endeavors, leading by example and to sustain and provide for their success. The credit for the success is shared by the team without particular focus on the leader's role.

vi. Adaptive-an "as appropriate" use of different styles of leadership that assumes the leader has the skills needed for each style. This requires 
expertise in emotional intelligence and the ability to know the leadership group that supports the leader.

vii. Integrative-also called the thoughtful leadership style by Jim Fisher. The leader also manages, engages, and directs in this model. A matrix approach to problems allows the leader to understand where to place effort at any particular time. The leader actually leads from the middle as they identify the most appropriate behavior at the time.

viii. Authoritative-based on the power of the position. This is usually a formal transactional type leader with clear structure that must be followed to achieve success.

ix. Influential-as the leader grows in familiarity with the members of the institution or team, the ability to guide the members in their choices and make changes in the culture increases as trust increases within the group. There is no hierarchical, financial, or authoritative component to this type of leadership.

x. Collins "Level 5" selfless-the leader becomes less of a factor in the decision making and the creditreceiving process because the team becomes the most important part of the success, similar to the shepherd approach.

(e) Reading resources categories are not limited to only "leadership" books. The broad subject material should address every aspect of leadership function.

i. Leadership function

ii. Emotional intelligence

iii. Conflict resolution

iv. Negotiation skills

v. Organizational structure and culture

vi. Team selection, building, and management

vii. Motivational skills

viii. Quality measures and continuous improvement

ix. Business experience

$\mathrm{x}$. Review articles.

(f) The references ${ }^{1-13}$ include a list of authors who have published leadership-related books. There are only two authors in this list who refer to leadership in surgery (Kibbe and Boyatzis). The basic principles mentioned in the areas of military leadership (McChrystal et al) and business (all the rest) can be found to apply to medicine and surgery. This group of authors makes it easier to refer young and old faculty members to a reading list which will keep interest and provide organized approaches to leadership with multiple different perspectives. The single author with the most experience in teaching emotional intelligence is Goleman. His text on emotional intelligence is a good place to start reading because almost every other author refers to the need for emotional intelligence as a prerequisite for learning and practicing leadership.
4. Practice

(a) Create a leadership culture at the top of the department or division

i. Assign a vice chair of faculty development and leadership. A commitment to leadership is more visible when a person wears the title and has a specific job to do around the culture of leadership. Ideas for training and opportunity creation should be the assignment for the individual.

ii. Create a functioning senior leadership group for advice and idea formulation. Senior leadership is not a formal agenda-driven meeting but a freeflowing discussion around topics that bubble to the surface and end up as emphasis points during division chiefs' meetings. The senior leadership group can support the chief and keep the chief grounded at the same time, since none of the senior leaders has a reason to withhold advice, criticism, or feedback from the chief.

iii. Establish a formal "leadership culture plan" by putting names to jobs. As names go on paper next to an assignment, the thought process for that objective takes on a reality that forces consideration of alternatives, personal desires of the team member, and best fit of job to team member based on personality and experience.

(b) Report

i. Written reports on the culture of leadership should be submitted to the monthly division chiefs meeting for discussion and support for the program in each specialty of the department.

ii. Submit results of leadership training to the departmental annual report and tell the supporters of the department that the leadership culture exists. The response to a formal program can lead to increased referrals for excellent students, residents, and faculty to the department and possibly an increase in patient referrals based on reputation of the members of the department.

iii. Reporting the leadership assignments to the institution indicates that there is a growing source for leaders in the department of surgery ready to serve the institution. The institution then serves as a mechanism for rewarding the efforts of the member of the department who dedicates themselves to growing as a leader.

iv. Action items at the senior leadership level form the basis for committee assignments. Since the senior leaders of the department have insights that address the issues of every aspect of the department, it is important to take advantage of these ideas and discussion points to create a strategic plan for the department. 


\section{How?}

The practical steps of establishing a culture of leadership in a department of surgery can occur in any order. The only absolute initial requirement is for the chief to embrace and declare support for the concept of creating a leadership culture. After this pivotal event, the process can flow in many different directions.

1. Constant emphasis on the culture of leadership by department leadership brings the focus of the membership in line with the idea. There needs to be a shared vision throughout the department to make this a sustainable effort.

2. Develop a program to establish a culture of leadership, agreed upon and supported by the governance council of the department. Specific plans are important to keep all in alignment.

3. Assign the vice chair of faculty development to make leadership position recommendations to the governance group. The chief is sometimes not the best person to identify potential leaders within the department due to limited exposure to daily behavior.

4. Develop a leadership curriculum with a psychology director, surgical education director, or vice chair of faculty development which can be delivered in multiple settings to individuals at multiple levels of experience. The seminar setting is very appropriate for faculty at all levels but may not be as effective for trainees who are potentially intimidated by a group discussion. In those circumstances, a didactic format with opportunity for discussion or moderator led panel may be accepted.

5. Establish a curriculum focused on the levels of experience possessed by the participants.

(a) Students and residents require basics of emotional intelligence and leadership concepts to understand the implications of their own daily behavior on their potential for leadership in their current setting. They will come to understand the implications on their future decisions and applications for graduate and postgraduate training.

(b) Junior faculty may not realize the need for leadership training until offered a monthly seminar specifically about leadership available opposite regular department educational conferences. This approach does not add to the burden of time demands on the junior faculties who have families and activities of daily life requirements. Leadership skills and options for approaches to daily leadership situations can enhance performance during patient care and while participating at a committee meeting.

(c) Mid-level and senior faculty who are committed to leadership positions may benefit more from classes at an offsite advanced learning institution which reduces the distractions of daily practice and institutional commitments. A mixture of leadership and business management skills training will lead to a comfort level in decision making that should improve performance in their home institution. Department, institution, or foundation funds need to be available to support this effort.

6. Develop a reward system within the department to entice junior faculty to attend a monthly seminar on leadership. While certificates are worth only the price of the paper they are written upon, they indicate a level of participation that indicates leadership potential. As the certificates appear on faculty walls, their value will rise. This becomes a reward to upwardly mobile individuals in the department. As a result, others will recognize this as a reward for their commitment to leadership education. This can only succeed when assignment to leadership positions is tied to prior participation in the course. Intradepartmental recognition for completion of the program will guarantee the value of the education.

7. Develop a mentorship program at all levels of the department with emphasis on resident mentoring and using leadership trained individuals as mentors. The mentor program must be maintained by an assistant program director in the residency program for it to be successful. Quarterly reports of meetings between resident and faculty mentor are key to the success. All mentors must receive short training in mentorship based on one of several publications within surgery that deal with the specific needs for surgical residents in training. Faculty mentorship can also be beneficial and can be established with or without departmental structure. Ayearly lunch with junior faculty has helped me as chief to understand the goals and desires of the newest faculty members over the years.

8. Provide opportunities for leadership for newly educated faculty. It is helpful to keep a list of those opportunities that need appointments and the corresponding list of faculty members that qualify. If the leadership position can be rotated through various faculties on an intermittent basis, it is helpful to keep track of the performance by each person who has been in the position. A required report from the leader is helpful to accomplish this process. As years pass and the junior faculty member grows into a senior leader, it will be important to track the service for that individual in their curriculum vitae as a record of service and documentation of team membership and leadership function.

9. Discuss and communicate metrics of success for practicing leaders. It is often confusing for new leaders to understand how their performance as a leader is measured. Personal success should not be judged in a vacuum since other people have contributed to the outcome. Team success reflects positively on the leader, unless the leader professes that the outcome was due only to leader participation. "Reflected light" from the success of others should become the goal of any successful leader. Once this concept is understood, true leadership begins.

10. Encourage societal membership and leadership positions by including these events in promotion criteria. A focus outside of personal surgical practice brings a new recognition of opportunities to improve the overall care of patients and broadens the world view of the surgeon. This is a benefit to 
the institution and department and should be supported with travel funds to send representatives to national and regional societal meetings. Time away from practice should not be compounded by requiring the faculty member to pay their own way to a meeting. The justification for travel should include presentation of research material or participating in national committee business, all of which provide exposure for the home institution. The higher a faculty member rises in the national society, the greater the exposure for their home institution and the more likely the faculty member will bring home leadership skills to benefit the department.

11. Utilize innovative ideas as opportunities for individuals to create and lead a program. Not all leadership is experienced in an existing structure. In fact, much of leadership is exhibited in the setting of innovation where no structure exists. The establishment of a program, process, or project requires an elevated level of leadership to be successful. While junior faculty often start programs as a part of their recruitment to an academic position, the learning about leadership that takes place is phenomenal. Support during this time is essential and the freedom to make mistakes during that time is essential. Formal training in leadership during this time will be very important and provides a practical example for application in real time. The lessons learned then become ingrained.

12. Utilize national organization leadership training available for members of the specialty society. The American College of Surgeons (ACS) has the most readily available leadership training for surgeons. It is important to send potential leaders to the ACS Spring Leadership summit in Washington, DC and assigned leaders within the department of the ACS surgeons as leaders conference in Chicago, which provide networking opportunities and exposure to the leaders of surgery in the United States. This exposure to the ideals of leadership can change a career as the individual sees what could be.

\section{Conclusion}

This description of starting a leadership training program is based on the practical implementation of a leadership culture in our training program at Baylor University Medical
Center in Dallas. It is the result of 5 years of learning about leadership within the department of surgery professionalism emphasis taught by Dr. Anthony Picchioni. His experience as a professor of psychology at Southern Methodist University and a leadership consultant for businesses has added greatly to the ability of our department to beginning our leadership culture. A focus on leadership enhances every aspect of departmental function and strengthens our ability to attract outstanding individuals to our department at trainee and faculty levels. It is our desire to provide unlimited opportunities for success for all who interact with our department.

\section{Conflict of Interest}

None declared.

\section{References}

1 Collins J. Good to Great. New York, NY: Harper Collins Publishers Inc; 2001

2 Wageman R, Nunes D, Burruss J, Richard Hackman J. Senior Leadership Teams. Boston, MA: Harvard Business Review Press; 2008

3 Lencioni P. The Ideal Team Player. How to Recognize and Cultivate the Three Essential Virtues. Hoboken, NJ: Jossey-Bass, a John Wiley \& Sons, Inc. imprint; 2016

4 Maxwell JC. Developing the Leader Within You. Nashville, TN: Thomas Nelson; 1993

5 Miller M. Leaders Made Here. Oakland, CA: Berrett-Koehler Publishers, Inc.; 2017

6 Kotter J, Cohen DS. The Heart of Change. Boston, MA: Harvard Business Review Press; 2002

7 Cialdini R. Pre-Suasion. A Revolutionary Way to Influence and Persuade. United Kingdom: Random House Books; 2016

8 Fisher J. The Thoughtful Leader. A Model of Integrative Leadership. Toronto, Buffalo, London: University of Toronto Press; 2016

9 Goleman D. Emotional Intelligence, 10th ed. New York, NY: Bantam Dell, A Division of Random House, Inc.; 2006

10 Kibbe M, Chen H. Leadership in Surgery. Switzerland: Springer International Publishing AG; 2015

11 McChrystal S, Collins T, Silverman D, Fussell C. Team of Teams. New Rules of Engagement for a Complex World. New York, NY: Penguin Random House LLC; 2015

12 Boyatzis R, McKee A. Resonant Leadership. Boston, MA: Harvard Business School Press; 2005

13 Richard Hackman J. Collaborative Intelligence. Using Teams to Solve Hard Problems. Oakland, CA: Berrett-Koehler Publishers, Inc.; 2011 